\title{
EL IMPACTO SOCIAL EN LAS EVALUACIONES DE IMPACTO AMBIENTAL: SU CONCEPTUALIZACION Y PRACTICA
}

\author{
Mercedes Pardo
}

Universidad Pública de Navarra

\section{INTRODUCCION}

La Evaluación de Impacto Ambiental (EIA) es un instrumento de gestión medioambiental dirigido a la evaluación de nuevos proyectos de desarrollo (públicos y privados), que se viene aplicando en muchos países desde su implementación por primera vez en Estados Unidos, en 1968, y de reciente obligación en España (1988) como consecuencia del requerimiento comunitario (Directiva 85/337). El análisis del impacto social de estos nuevos proyectos se realiza en el marco de estas EIA.

En Europa la experiencia en este campo es bastante reciente, pero se cuenta con más de un cuarto de siglo en Estados Unidos, seguido de Canadá (1973) y de otros países en todos los continentes, lo que está permitiendo una evaluación de resultados.

Muy en síntesis, se puede decir que, a pesar de la intensa actividad en los ámbitos de investigación y gestión llevada a cabo, este campo todavía adolece de problemas importantes. Los más destacables se refieren a las limitaciones en la regulación legal y sus procedimientos, la falta de control y cumplimiento de las medidas correctoras y complementarias obligatorias, la aprobación de proyectos dudosos desde el punto de vista medioambiental, la deficiencia de los instrumentos teóricos y analíticos que tiene el campo, el escaso interés social y de participación pública. No obstante las anteriores precauciones, la Evalua- 
ción de Impacto Ambiental se considera como uno de los instrumentos más interesantes a nivel mundial en los que se puede basar la gestión de una política medioambiental.

En España, después de seis años de su entrada en vigor, no se ha realizado ningún diagnóstico amplio de la situación, aunque sí se coincide por parte de los diversos sectores implicados (Administración, profesionales, organizaciones sociales) en su deterioro profundo. Las razones para esta situación son varias: la falta de una política medioambiental definida, de manera que la EIA es, sobre todo, una imposición comunitaria; el marco económico competitivo mundial, en el que se justifica la priorización absoluta del desarrollo económico sobre cualquier otra consideración medioambiental ${ }^{1}$; escasos recursos económicos y humanos para la administración medioambiental'; falta de formación profesional para el campo específico de la EIA, de manera que los equipos de trabajo están formados, sobre todo, por licenciados especializados en las distintas disciplinas, pero que carecen de formación general en el campo ${ }^{3}$.

Todos los puntos anteriores se retroalimentan en la medida en que la Administración medioambiental no hace - o no puede hacer- unos controles serios de la calidad técnica de los estudios de impacto, ni un seguimiento del cumplimiento del Condicionado Ambiental.

Finalmente, otro aspecto importante para explicar la situación descrita es el escaso interés sociopolítico de las EIA para organizaciones sociales en general y para las organizaciones ecologistas en particular ${ }^{4}$, cuya participación en este campo ha estado básicamente orientada a aquellos temas especialmente notorios 5 .

En ese contexto, este artículo analiza la conceptualización del impacto social dentro de las Evaluaciones de Impacto Ambiental en España, y se basa

${ }_{1}$ Es archisabido la necesidad de sacar el Plan Nacional de Carreteras rápidamente, lo cual supuso, entre otras cosas, la negativa de la CEE a financiar 40.000 millones de pesetas por razón de haberse obviado los Estudios de Impacto Ambiental obligatorios.

${ }^{2}$ La Administración Central, por ejemplo, ha sufrido una acumulación de proyectos pendientes de declaración oficial, con retrasos a veces de un año, y en cualquier caso una media de seis meses parece bastante real.

${ }^{3}$ Con motivo de la reforma universitaria se empiezan a ofrecer asignaturas de temas medioambientales en algunas facultades. La formación en EIA producida hasta ahora ha sido: cursos de formación para funcionarios organizados por las administraciones durante varios años; cursos específicos organizados por organizaciones profesionales (el único curso que se tenga noticia por la autora sobre Evaluación del Impacto Social en la EIA se impartió por el Colegio Oficial de Políticas y Sociología en 1991); algunos Master más recientemente (el pionero fue el organización por la Universidad Politécnica, Escuela de Ingenieros de Montes). Para aquellos interesados en profundizar en las necesidades por países de la CEE, véase N. LEE y C. WOOD, «EIA Training and Research in the European Communities», Working paper, Centre for EIA, University of Manchester, 1991.

${ }^{4}$ Hay que aclarar que las organizaciones ecologistas españolas son mucho más débiles que lo que pueden ser las norteamericanas, las alemanas o las francesas, por poner algunos ejemplos.

5 Por ejemplo, el embalse de Riaño, el embalse de Itoiz, el Tren de Alta Velocidad MadridSevilla, entre otros. 
en el análisis de la legislación y guías metodológicas y en su práctica a través de los estudios concretos realizados, todo ello en el marco más amplio teórico del significado actual del impacto ecológico y social.

El análisis concreto de casos, hecho por la Comisión de Sociología del Medio Ambiente del Colegio de Sociólogos y el Colegio de Biólogos ${ }^{6}$, es el primero de este tipo que se realiza en España sobre las $\mathrm{EIA}^{7}$, y creemos que sus resultados son bastante sintomáticos del enfoque y concepto del impacto ambiental que tiene la Administración ambiental española. Este artículo es parte de una investigación más amplia que ha dado lugar a otros artículos, por lo que algunos de los puntos que aquí se presentan ya han sido tratados por la autora $^{8}$, pero se incorporan aquí para una mejor comprensión autónoma del texto que aquí se presenta.

\section{EL MEDIO AMBIENTE, EL IMPACTO ECOLOGICO, EL IMPACTO SOCIAL}

La utilización del concepto de medio ambiente es relativamente reciente ${ }^{9}$ y, además, responde a una interpretación social del medio con el que interactuamos como especie humana. Se formula a partir de una determinada valoración y conocimiento de la realidad, que básicamente consiste en que hay que preservar el medio biofísico en el que vivimos para seguir perpetuándonos como especie dominante. Recientemente está tomando peso una línea de pensamiento sobre los «derechos» de otras especies en sí mismas a existir, con independencia de nuestro mayor o menor interés en ellas ${ }^{10}$, pero el concepto de especie dominante es el preponderante.

De esa interpretación global del medio ambiente en el modelo económico dominante se desprenden dos aspectos importantes ${ }^{11}$. Por una parte, se basa en

${ }^{6}$ Este análisis se ha hecho en la investigación «La Evaluación de Impacto Ambiental y el desarrollo sostenible, Fase I» (Mercedes Pardo, Alberto Fraguas, Pilar Galindo, Rafael González, Enrique Ramos, Cándido Robledo), Plan Regional de Investigación de la Comunidad de Madrid, 1993.

${ }^{7}$ La CE encargó un estudio-diagnóstico al Center for EIA (Centro para la Evaluación del Impacto Ambiental), de la Universidad de Manchester, para el seguimiento de las EIA en los países comunitarios, pero no con carácter discrecional y además muy global.

${ }^{8}$ Véase Mercedes PARdo, «La Evaluación de Impacto Ambiental en España: conceptualización y práctica», pendiente de publicación.

9 Nos estamos refiriendo al desarrollo de la concienciación sobre los problemas del medio ambiente en la década de los sesenta, porque, evidentemente, todas las culturas y sociedades anteriores han tenido su conceptualización del medio ambiente en el que han vivido.

${ }^{10}$ La Escuela de Ecología Profunda es una buena introducción al tema. Véase el filósofo noruego Naess (1973), que fue el primero en utilizar el término, y Tobias (1984), entre otros, y más recientemente Nash (1989).

${ }^{11}$ Una primera versión de esta reflexión se preparó con motivo de una ponencia presentada por Pilar Galindo y Mercedes Pardo sobre el impacto social de las obras públicas para unas Jornadas sobre el Paisaje organizadas en Segovia en 1992. 
la creencia de que la especie humana no está sometida a restricciones por la naturaleza ${ }^{12}$ y que los problemas que van apareciendo pueden irse resolviendo con los avances tecnológicos y la gestión (por ejemplo, rehabilitación a través de la tecnología; tecnología adecuada). Por otra parte, está la propia definición que se hace de los problemas medioambientales, en términos de lo que constituye un problema medioambiental o de lo que no, ligado a la estructura de poder social (diferentes definiciones según: países desarrollados-países en desarrollo, ciudades-áreas rurales, mayorías-minorías...).

El surgimiento del medio ambiente como objeto de interés no es casual. Se desarrolla y comienza a debatirse socialmente a través de los medios de comunicación en un momento en que está en crisis el modelo dominante de gestión y de utilización de los recursos naturales en general y energéticos en particular, que son escasos y agotables ${ }^{13}$. Los efectos de las políticas y concepciones económicas se han vuelto como un boomerang sobre sí mismas en lo relativo a los problemas medioambientales, con consecuencias no previstas inicialmente ${ }^{14}$. Pero, es más, la crisis medioambiental abierta puede estar apuntando no solamente al modelo económico como tal, sino incluso al propio concepto de modernización (Toledo, 1991) y, por tanto, al modelo de desarrollo en el que la propia evaluación ambiental se basa.

El debate social de la crisis ambiental gira alrededor de cómo resolver el problema. Dentro de las concepciones dominantes, lo que básicamente se pretende es integrar la variable ambiental en el proceso de gestión y utilización de los recursos naturales y, por tanto, del medio que soporta el desarrollo tecnológico y económico $^{15}$. Se introducen correcciones ambientales (depuración de aguas, repoblaciones forestales, control de inundaciones, reducción de las emisiones contaminantes a la atmósfera), pero no se modifica el modelo económico-social, por lo que, según algunos autores (Schnaiberg, 1980), las correcciones no pueden resolver las contradicciones de fondo, limitándose a resolver pequeños problemas o problemas a medias ${ }^{16}$. En ese sentido es cuando el impacto medioambiental, como concepto y como realidad, adquiere su mayor relevancia social, puesto que pone de manifiesto que las mejoras que el desarrollo económico proporciona a la sociedad no son tan ventajosas como se suponía.

12 Catton y Dunlap (1978), pioneros de la Escuela de Sociología Medioambiental norteamericana, lo calificaron como el Paradigma del Excepcionalismo Humano.

13 Especialmente a partir de la crisis del petróleo de 1973-1974.

14 Martínez-Alier (1987) y Daly (1991) han analizado el tema y forman parte del movimiento de economía ecológica.

15 Desde las teorías económicas basadas en el mantenimiento del sistema se están dando respuestas interesantes que abarcan desde la ampliación de las posiciones neoclásicas hasta el nuevo movimiento de economía ecológica.

${ }^{16}$ Es interesante el análisis crítico de sus veinte años de gestión medioambiental que hace la EPA (Agencia de Medio Ambiente de EE.UU.), donde concluye que muy pocos de los problemas medioambientales se han resuelto, algunas de cuyas soluciones han significado "quitar la contaminación de aquí para ponerla en otro sitio» - p. ej., residuos-, y otros nuevos y desafiantes problemas están apareciendo — - ej., biotecnología-. 
El impacto medioambiental tiene también otra característica de unas consecuencias enormes. Cuando se habla de medio ambiente se introduce una valoración de cómo debe ser la naturaleza y la sociedad que convive-utiliza-disfruta con ella. Esa es la base fundamental del debate social. Pero lo que aquí interesa poner de manifiesto es que en esa valoración subyace el concepto de ecosistema (Margalef, 1968; Morin, 1980) como conjunto de las interacciones en el seno de una unidad geofísica determinable que contenga diversas poblaciones vivientes y que constituye una unidad compleja de carácter organizador o sistema.

De esta manera, con la asunción del concepto ecosistémico de medio ambiente, el divorcio histórico existente entre sociedad y naturaleza, como dos bloques antinómicos ${ }^{17}$, ya no es posible. Los seres humanos formamos parte de la naturaleza, de la cual dependemos, al menos en términos de energía y recursos básicos, para nuestra subsistencia, y a la cual impactamos profundamente a través de nuestra cultura y organización social (López Rodríguez, 1984). En nuestras sociedades actuales, el modelo de relaciones sociedad-naturaleza es bastante complejo, pero ello no cambia el principio básico de interrelaciones ecosistémicas. Esta definición integrada está produciendo una necesidad de reconceptualizar el impacto social.

Aparece entonces el otro elemento configurador del concepto de impacto medioambiental, el de sostenibilidad. El concepto de desarrollo sostenible ha ido evolucionando desde un enfoque exclusivamente biofísico a una definición más amplia que incluye objetivos de sostenibilidad social (Tolba, 1984). Para la mayoría de los autores, y con independencia de que se consideren además aspectos sociales o no, el término desarrollo sostenible es intercambiable con ecológicamente sostenible o desarrollo medioambientalmente sano. Pero, a veces, el desarrollo sostenible se interpreta como "crecimiento sostenido", "cambio sostenido", o, simplemente, el término "desarrollo» se usa en vez de «éxito» (Lele, 1989).

Lo que aquí interesa destacar, en cualquier caso, es la dimensión histórica y temporal hacia el futuro que el concepto de sostenibilidad introduce en el impacto medioambiental ${ }^{18}$. El derecho de las generaciones futuras a un medio ambiente adecuado está planteando un conflicto moral que no hay duda va a producir repercusiones económicas considerables (Norgaard, 1989).

El concepto de impacto ambiental ha ido así cambiando a lo largo de estos años de polémica sobre el tema, que en síntesis iría desde un enfoque casi exclusivamente biofísico y reduccionista en las políticas conservacionistas de los sesenta y setenta, a una concepción del medio ambiente más amplia e integrada, en donde lo social juega un papel fundamental en la configuración de lo que se viene a llamar "problemática medioambiental».

17 Benton (1991) plantea que se está produciendo un desafío a los dualismos que están por debajo de las tradiciones teóricas dominantes: cuerpo/mente, biología/sociedad, causa/significado, naturaleza/cultura...

${ }_{18}$ Especialmente interesante es la incorporación del concepto de sostenibilidad como criterio en las EIA, en las directivas y recomendaciones del Banco Mundial. 
De esta manera, el impacto ambiental es el resultado de las interrelaciones del impacto ecológico y del impacto social. Esas relaciones son múltiples y complejas, pero, en cualquier caso, la conexión es inmediata en la medida en que la regulación del impacto ecológico deviene social en sus consecuencias y es la sociedad, en definitiva, quien interpreta ambos y les da contenido.

\section{EL MODELO DESARROLLADO}

El análisis del modelo imperante en España de la EIA se hace en esta investigación sobre tres aspectos fundamentales: la legislación aplicable, los modelos metodológicos propuestos y la práctica concreta que se viene desarrollando, cogiendo como ejemplo las obras públicas.

De forma muy resumida, la EIA concreta de un proyecto de desarrollo propuesto consta de los siguientes aspectos:

1) Presentación de la Memoria-Resumen del proyecto ante el órgano ambiental competente.

2) Inicio de consultas previas y, con ellas, indicaciones para el Estudio de Impacto Ambiental.

3) Estudio de Impacto Ambiental.

4) Período de Información Pública y Alegaciones.

5) Formulación de la Declaración de Impacto Ambiental y condiciones que ésta impone, y Seguimiento y Control del proyecto desde el punto de vista ambiental.

Lo social en este modelo tendría aquí dos representaciones. Por una parte, lo que serían los aspectos participativos de la población en el proceso de definición y decisión sobre los impactos y, por otra, el análisis del impacto social del proyecto. Esta es la división formal, correspondiendo el primer aspecto a los puntos 2 y 4, mientras que el segundo - el análisis del impacto social- se haría en el 3, el correspondiente al estudio técnico propiamente dicho. En la práctica, esa división no es tan estricta y los dos aspectos resultan influidos unos por otros. Es controvertido cómo situar el análisis del propio objetivo social del proyecto de desarrollo propuesto y su ubicación en políticas territoriales y sociales más amplias que, aunque exigirían su evaluación en momentos anteriores, forman también parte de la evaluación social que se hace en el momento del proyecto.

Por lo que respecta a los procesos de participación pública, éstos, virtualmente no se dan. Salvo excepciones — por lo general, cuando un proyecto ha llegado a ser notoriamente conflictivo-, la participación pública es muy pobre. Este es, probablemente, uno de los problemas más graves, ya que la filosofía preventiva de la EIA necesita precisamente de ese input, aparte de otras consideraciones de derecho. El estudio, pues, se va a centrar aquí en el análisis del impacto social. 
La legislación naciona ${ }^{19}$ establece el marco general, obligando a la evaluación de los efectos previsibles directos e indirectos del proyecto sobre la población ${ }^{20}$, la flora, la fauna, el suelo, el aire, el agua, los factores climáticos, el paisaje y los bienes materiales, incluido el patrimonio histórico-artístico y el arqueológico; alude a la elaboración de las medidas para reducir, eliminar o compensar los efectos ambientales negativos derivados del proyecto propuesto, incluyendo posibles alternativas existentes a las condiciones inicialmente previstas, y exige la presentación de un resumen del estudio y conclusiones en términos fácilmente comprensibles, con la intención de que sirva para el período de información pública; exige también la indemnización por los daños y perjuicios ocasionados.

El Reglamento de aplicación amplía la estimación de los efectos al estudio de la estructura y función de los ecosistemas presentes en el área previsiblemente afectada..., relaciones sociales y condiciones de sosiego público tales como ruidos, vibraciones, olores y emisiones luminosas y la de cualquier otra incidencia ambiental derivada de su ejecución; determina también que se indiquen las interacciones ecológicas y ambientales claves, incluyendo un estudio de diagnóstico comparado de la situación ambiental actual y futura para cada alternativa examinada. Se añade que se especifiquen los procedimientos utilizados para conocer el grado de aceptación o repulsa social de la actividad, así como las implicaciones económicas de sus (del proyecto) efectos ambientales.

Desde el punto de vista de cómo se conceptualiza el impacto ambiental ${ }^{21}$, se produce una mejora considerable en este Reglamento al convertir el Estudio de Impacto Ambiental en un diagnóstico de los procesos generados en el medio ambiente en general, y específicamente en el medio social afectado, mientras que la interpretación del Decreto legislativo es más descriptiva de los diferentes elementos concurrentes, pero escasamente de las relaciones entre ellos.

Sin embargo, la evaluación de los impactos está muy enfocada a determinar una gradación negativa del impacto (compatible, moderado, severo y crítico), desplazando con ello los efectos positivos, lo que no permite evaluar el impacto social en su complejidad, ni permite otro tipo de enfoques diferente al estrictamente defensivo. En la práctica, muy a menudo ocurre que se utilizan los beneficios sociales potenciales del proyecto como moneda de cambio para su aceptación.

19 RDL 1302/86, Reglamento de Aplicación 1131/88, básicamente con algunos artículos de otras leyes. La legislación autonómica desarrollada posteriormente no se incluye en este análisis, pues básicamente no cambia el modelo que se plantea en la nacional; no obstante, para un análisis más detallado de esta última, véase M. PARDO et al., "La EIA y el desarrollo sostenible, Fase I», op. cit.

${ }^{20}$ La cursiva es mía.

${ }^{21}$ Para una crítica abarcando otros muchos aspectos, véase José AlLENDE LANDA (1990), «La Evaluación de Impacto Ambiental. Marco de referencia y aspectos relevantes a debatir», Ciudady Territorio, 83-1; Mercedes PARDO, «Las Evaluaciones de Impacto Ambiental en España: conceptualización y práctica», pendiente de publicación. 
La evaluación del impacto de las obras públicas, por ejemplo, es algo más amplio que la simplificación impacto negativo ecológico-impacto positivo social. Cualquier obra pública pretende un beneficio social - al menos en teoría-, pero también comporta efectos negativos. Ambos aspectos hay que analizarlos cuidadosamente con el fin de evaluar el nivel de viabilidad social y ecológica de un proyecto y las posibilidades de minimizar los efectos negativos y de potenciar al máximo las oportunidades de mejora que se planteen. El propio objetivo social que pretende dicha obra pública es algo a evaluar no solamente en sí mismo, sino también estudiando si hubiera otras alternativas y/o modos menos impactantes que pudieran conseguir los objetivos sociales propuestos.

Un ejemplo de lo anterior serían alternativas a un gran embalse que podría consistir en una combinación de varios embalses más pequeños, políticas de conservación del agua, mejoras tecnológicas de los regadíos para minimizar el agua necesaria u otras que, muy probablemente, saldrían si hubiera una actitud abierta a la consideración de alternativas. Es ilustrador, al respecto, el cambio de política de una de las empresas eléctricas más importantes de California (PG\&E), que ha optado por potenciar la conservación de la energía entre sus clientes en vez de construir nuevas centrales eléctricas — costosas y problemáticas ecológica y socialmente- Algunos de los cambios que se están produciendo en el Plan Hidrológico Nacional van en esa línea de sustituir parte de los embalses proyectados de nueva construcción por políticas de disminución de la demanda, en especial la conservación y el ahorro en el consumo del agua.

En definitiva, la caracterización que se hace del impacto resultante de diversos efectos de distinto signo, magnitud, duración, etc., sobre la población afectada, o la economía, o la cultura, o la organización social, no puede evaluarse simplemente en términos de compatibilidad o en distintos grados de negatividad, como plantea el Decreto legislativo. En otros casos, tampoco puede establecerse a priori si será positivo o negativo. El tema es más complejo.

Supóngase un embalse proyectado para proporcionar agua para implantar regadíos en las tierras que están aguas abajo de la futura presa. De entrada, parecería prudente que la legislación diera margen a evaluar la viabilidad del proyecto teniendo en cuenta que muchos embalses fueron proyectados hace años en condiciones socioeconómicas diferentes a las actuales y que existen políticas comunitarias de eliminación de excedentes agrícolas, fomentando el abandono de tierras de cultivo, incluyendo regadíos, por razones varias. Pero, suponiendo el sentido económico de la obra, los efectos sociales de la misma pueden llegar a ser muy diversos. Los propietarios y arrendatarios de las tierras que vayan a regarse estarán, en principio, a favor del proyecto. Sin embargo, los propietarios o arrendatarios de las tierras a inundar no tendrán una única visión. Si cultivan sus tierras no quedarán conformes con una expropiación, por muy bien pagada que esté. Si se les propone un cambio a una zona próxima, quizá se consideran compensados, pero puede que aun así se sientan perjudicados. Si son arrendatarios, seguramente estarán en contra de no tener dere- 
cho a expropiación aunque se les compense económicamente. También hay otros factores, como son la inundación de sus viviendas y los realojos consiguientes, tener que dar grandes rodeos para desplazarse hasta las parcelas de cultivos, suponiendo que se restituyan la mayor parte de las comunicaciones, etcétera. De este modo, el impacto sobre los que usufructan o tienen en propiedad las tierras de cultivo tiene demasiados matices para ser reducido a la categorización del Decreto y, desde luego, siempre depende de la negociación.

Véase a través de estos simples ejemplos la carga valorativa que impone la legislación al exigir al estudio de impacto el diagnóstico en términos tan rígidos como los indicados más arriba sobre niveles de compatibilidad. Más útil y acorde con la filosofía de la evaluación medioambiental sería un enfoque dirigido, sobre todo, a producir una información organizada, en donde se pongan de manifiesto las diferentes perspectivas y matices que se produzcan en el proceso social de valoración, como soporte del proceso de negociación y de toma de decisiones.

Por otra parte, las Guías Metodológicas elaboradas por la Dirección General del Medio Ambiente (actualmente Secretaría de Estado) ${ }^{22}$ han tratado de desarrollar un modelo con el que abordar los Estudios de Impacto Ambiental. Desde el análisis de su procedimiento y puesta en práctica se observan deficiencias que se traducen en los Estudios de Impacto Ambiental concretos llevados a cabo.

Se expone a continuación un esquema sintetizado de las fases que caracterizan a un Estudio de Impacto Ambiental:

- Análisis características del proyecto - Identificación y Valoración

- Análisis características del medio

- Medidas Correctoras

La primera consideración a destacar es que estas guías están caracterizadas por una visión estática y técnica del proyecto, sin considerar los condicionantes económicos, tecnológicos y políticos que intervienen en la justificación del proyecto y que constituyen un argumento fundamental en la determinación de las alternativas al proyecto y del impacto social del mismo.

Junto con lo anterior, otra crítica central de estas guías es que el enfoque del contenido de los estudios a realizar se dirige a la descripción y elaboración de índices, base sobre la cual es prácticamente imposible una previsión y valoración seria de los impactos. Principalmente se dirige a la recolección de datos secundarios con escasísima utilización de investigación primaria sobre la reali-

22 Seguimos aquí refiriéndonos a las Guías Metodológicas elaboradas por el MOPU (actual MOPTMA) en relación a la legislación de la EIA. 
dad socioeconómica en la que va a estar inmerso el proyecto, tan necesaria para la valoración del impacto social previsible.

La identificación y valoración de los impactos, las medidas correctoras y el plan de vigilancia, que son los tres aspectos centrales de estos estudios, se analizan con una visión atomizada de los procesos. Los impactos se analizan basándose principalmente en la técnica «matriz de impactos», en donde se listan, por un lado, los elementos del proyecto y, por otro, los elementos componentes del medio, para nuestro análisis del medio social; las medidas correctoras que se consideran como viables en relación a lo social es casi exclusivamente la indemnización económica; y los planes de vigilancia son genéricos y globales, sin mayor fuerza ${ }^{23}$.

Con esta estructura metodológica lo que se produce realmente es una validación de facto del proyecto, dirigiéndose básicamente a la mitigación de las acciones del proyecto con efectos negativos y, principalmente, sobre el medio biofísico. Se traduce nuevamente la visión estática realizada en el análisis del proyecto, sin dar cabida a cuestiones como localización, nuevas alternativas o el mismo sentido del proyecto.

En el «haber» de estas guías está el intento de desarrollar una fase de inventario bastante exhaustiva (se incluyen, por ejemplo, en lo relativo al análisis del medio ambiente social, temas como el estudio del sistema territorial y los usos del suelo, el análisis demográfico y económico, el análisis de la estructura social y cultural, de la opinión pública, y otros). Sin embargo, al no haber desarrollado sus fases de diagnóstico y de identificación y valoración de impactos, no dan lugar a la evaluación social del proyecto.

\section{Estudio de casos}

Se va a centrar en el análisis de casos de proyectos de embalses, carreteras (autovías y autopistas), sobre los que la Dirección General de Política Ambiental ha emitido Declaración de Impacto Ambiental. Se basa en algunas consideraciones de la investigación indicada anteriormente, realizada por la Comisión de Sociología del Medio Ambiente del Colegio Oficial de Políticas y Sociología y la Comisión de Medio Ambiente del Colegio de Biólogos.

La Declaración de Impacto Ambiental (DIA) es el documento oficial, legal, publicado en el Boletín Oficial del Estado y vinculante en sus condiciones. La DIA tiene que reflejar todo el proceso de la Evaluación de Impacto Ambiental en todos sus aspectos: consultas previas, estudio de impacto ambiental, medidas correctoras, plan de vigilancia ambiental, proceso de participación pública. Tiene, finalmente, que establecer las Condiciones Ambientales del proyecto, es decir, la aprobación del proyecto sometida a aquellos aspectos medioambientales que tiene obligatoriamente que cumplir.

23 Para un análisis más detallado de estos aspectos, véase M. PARDO y colaboradores, «La Evaluación de Impacto Ambiental y el desarrollo sostenible», op. cit. 


\section{EL IMPACTO SOCIAL DE LOS EMBALSES}

Se emitieron un total de siete Declaraciones de Impacto Ambiental (DIA) de embalses hasta agosto de 1992. Veamos las conclusiones a las que llevan dichas declaraciones:

- Todas las Declaraciones emitidas sobre embalses establecen que éstos son medioambientalmente viables, bajo los requisitos expuestos en su declaración. Es decir, no se ha denegado ninguno de los permisos, ni se han planteado alternativas de localización o de tipo de presa.

- Sobre los objetivos y justificación para la construcción de estos embalses, en una de las Declaraciones no se describen, y del resto, tres son para laminar avenidas, dos para regadío y una para abastecimiento de agua potable.

- Solamente las últimas tres Declaraciones oficiales emitidas incluyen descripción del proyecto, resumen del Estudio de Impacto Ambiental y de las consultas y alegaciones públicas presentadas. En ninguna de ellas se expresa el grado de aceptación social del proyecto, a pesar de ser uno de los puntos importantes destacados en la legislación (RD 1131/88).

- Las alegaciones relativas a los perjuicios socioeconómicos no se tienen en cuenta o no se contestan debidamente por la Declaración Medioambiental. A lo sumo, en su caso, relega a una fase posterior el estudio de las repercusiones en la evolución de los cultivos y su productividad con motivo del regadío. En el resto de los casos, la afección a tierras a inundar de alto valor agrícola se contempla únicamente recomendando la reducción, en la medida de lo posible, de su ocupación.

- Para aquellos embalses que conllevan inundación de pueblos y tierras de cultivo o pastos, no se especifica debidamente el modo cómo se va a resolver dicha situación, dando por sentado que un traslado a otro pueblo de la comarca es suficiente.

- Los aspectos que más se tienen en cuenta son la reposición de caminos y de patrimonio histórico, o restos arqueológicos, pero, en el caso de los primeros, no se especifica en general cómo se va a hacer y si con ello se resuelve de la mejor manera posible el problema, o si queda reducida la accesibilidad.

- No se plantean medidas compensatorias o complementarias para actividades económicas de los pueblos directamente perjudicados, a excepción de un caso en el que se somete a un estudio posterior la posibilidad de regular usos recreativos en el embalse como un beneficio adicional.

Hasta ahí las conclusiones más destacables del estudio de casos, continuando una tendencia similar en los chequeados más recientemente. Pero veamos el tema en más detalle.

Existe una abundante literatura sobre el análisis del impacto social de las obras públicas, y en concreto de embalses, que coincide en que la identificación de impactos en el medio social no depende tanto del detalle con que se 
produzca la descripción a través de indicadores que, aunque siempre resultan válidos, no son suficientes. Lo más importante es el trabajo de campo que se realice, porque una parte fundamental del análisis del impacto es la valoración que hacen de ese impacto las poblaciones y los distintos colectivos sociales que van a verse afectados de una u otra manera.

Pero, es más, la literatura nos indica que uno de los problemas de la evaluación de impactos es la tendencia de los expertos - profesionales del campo y responsables de la Administración- a lo que Clarke (1990) denomina «la descalificación heurística», es decir, la tentación a cerrar los temas prematuramente. Una vez han dado una lista de impactos potenciales, los expertos tienden a asumir que está básicamente completa y fallan en muchos casos en reconocer posteriormente problemas potenciales que han omitido. Esto, unido a la presión por la cuantificación como validación de la objetividad de la valoración del impacto, produce la "tiranía de la precisión ilusoria». La investigación a posteriori de las EIA indica (Murdock, 1982; Bisset, 1987; Culhane, 1987) que incluso para los impactos más fácilmente cuantificables, como pueden ser los demográficos y los económicos, se da una media de error en las previsiones entre el 50-100 por 100 .

Pero veamos qué tipo de problemática tiene la definición de los efectos sociales directos o inducidos de la construcción de embalses dentro del campo de la Evaluación de Impacto Ambiental.

Una primera diferenciación que debería hacerse es identificar los colectivos que, teóricamente, van a verse beneficiados por la obra (regantes aguas abajo de la presa, poblaciones que van a utilizar el abastecimiento de agua, propietarios o arrendatarios que han visto inundadas sus tierras por las avenidas, etc.) y los que van a perjudicarse con ella. Entre éstos cabe hacer matizaciones: los directamente expropiados que sólo tienen sus tierras y no viven o no van a volver al pueblo y los que, además de tierras, poseen vivienda, que utilizan ya sea temporalmente o todo el año; los residentes en el pueblo o pueblos afectados aunque no se expropie su vivienda; los nacidos en el pueblo que han emigrado y después de algunos años han arreglado la casa familiar; los residentes en el valle que utilizan los caminos o carreteras actuales y van a ver cortada su accesibilidad.

Efectivamente, son bastantes los colectivos. Por otro lado, habría que tener en cuenta su edad y arraigo o desarraigo con la zona. Existe la tendencia a valorar con poco rigor los pueblos envejecidos que sólo en la época estival se animan con los «veraneantes» y en invierno languidecen. Evidentemente, no es lo mismo que un pueblo que mantiene su actividad y población durante todo el año, pero sí se debe tener en cuenta la tendencia cada vez mayor que tienen los pueblos españoles de revitalizarse estacionalmente y donde emigrantes del pueblo invierten sus ahorros en arreglar o construir su casa, manteniéndose la tendencia incluso por los nietos (no ha habido posibilidad de más generaciones). El impacto que puede provocarse es alto, incluso aunque sólo se afecte al camino de acceso rodado o aumenten las dificultades de vivir en el pueblo 
en época de crecidas por inundaciones o encharcamiento del camino. Los paseos por los caminos y rincones tradicionales del municipio también deben tenerse en cuenta, como elementos vertebradores muchas veces de la vida social del municipio.

Desde el punto de vista económico, habría que hacer nuevamente matizaciones. Los usufructuarios de las aguas de regadío suelen estar muy animados favorablemente hacia el proyecto, incluso aunque tengan que pagar un canon por el riego. Pero pocas veces se evalúa en esta fase del proyecto en qué medida van a beneficiarse realmente: ¿cuánta agua van a poder consumir con regularidad?; ¿qué cultivos son los propicios en función de la nueva situación del mercado europeo?; ¿qué abono debe emplearse?, en caso de salinidad de las aguas; ¿en qué medida va a ser rentable el riego?, y otras cuestiones como nivel de lavado de las parcelas, suspensión temporal del riego por salinidad inaceptable, etc. El exceso de medidas de seguridad en la construcción de la presa para corregir el riesgo de avenidas puede provocar nuevos desastres si viene una avenida que obliga a abrir el aliviadero o lo desborda.

Los que ven inundadas sus tierras, ¿consideran que la expropiación es un pago justo?, ¿prefieren una permuta de terrenos?, ¿dónde estarían dispuestos a que se les trasladasen sus propiedades? Si se traslada también la residencia, ¿se modifica negativamente su aceptación?, ¿están conformes con un traslado a otro pueblo o prefieren un asentamiento propio?, ¿se valora la ansiedad y estrés por el que van a pasar, sobre todo la población anciana?

Desde el punto de vista de los empleos que genera la obra, ¿cuántos empleos proporciona que sean realmente adecuados para la mano de obra que puede ofrecerse a nivel local?, ¿qué pasará cuando termine el proyecto?, ¿volverán a sus tierras o, si han sido expropiadas, emigrarán?, ¿qué consecuencias ecológicas van a producirse por el abandono de las tierras de cultivo y los usos tradicionales del territorio? Muchas veces el efecto del empleo se magnifica en cualquier caso, aunque dé trabajo a unos pocos o sólo se tenga en cuenta la demanda de alojamiento y manutención que generarán los obreros, la cual, además, se puede producir normalmente en pueblos mayores aguas abajo de la presa, no en los pueblos pequeños directamente afectados. Y, lo que es peor, persistentemente el empleo que va a dar la obra se utiliza como justificación de cualquier otra consideración social y ambiental.

Si se pretende aprovechar el embalse también para esparcimiento, deportes acuáticos y similares, también eso es normalmente considerado como algo positivo para la zona, pero los efectos de este uso a menudo son difícilmente evaluables — sobre todo si ha sido a expensas de perder tierras de valor agrícola o similares situaciones-y no revierten necesariamente en los vecinos, sino que el negocio suele favorecer a extraños que cuentan con más información e iniciativa.

En cuanto a las huellas culturales de edificios y restos arqueológicos, las medidas suelen encaminarse, en el mejor de los casos, a su traslado, siguiendo el criterio de los organismos responsables, y no siempre van al mismo lugar a 
donde se desplaza a los vecinos del pueblo, con lo que les queda la sensación de que se los han robado.

En resumen, los impactos sociales que genera la construcción de un nuevo embalse dependen fundamentalmente del modo en el que se hagan las cosas, incluyendo el momento en que se proyectó su realización, pasadas a veces varias décadas, y, sobre todo, el momento en que se tenga en cuenta a la población, los acuerdos a que se llegue y la amplitud de grupos sociales de afectados que participen en la negociación.

Pero ese proceso suele transcurrir independientemente de la Evaluación de Impacto Ambiental y, con toda seguridad, del Estudio de Impacto Ambiental. Por esa razón, en el proceso de participación pública las alegaciones no aparecen y el debate se concentra en el momento de la definición de la superficie expropiable, cuando el promotor contacta con algunos de los afectados y no lo hace con otros que también se consideran perjudicados o, al menos, implicados en el proceso.

Este desfase no ocurriría si realmente se introdujeran en el Estudio de Impacto Ambiental los objetivos claros del proyecto y su justificación detallada de los beneficios del mismo, así como la valoración del grado de aceptación social del proyecto, a través de los cuales debe definirse la identificación y valoración de impacto social y la definición de las medidas correctoras y compensatorias.

\section{EL IMPACTO SOCIAL DE LAS CARRETERAS Y AUTOVIAS}

Se produjeron un total de treinta y tres Declaraciones de Impacto Ambiental hasta la fecha estudiada (agosto de 1992), observándose en todas ellas la falta de una auténtica valoración del impacto social, que se confirma en los estudios recientemente chequeados.

- En las Declaraciones anteriores a 1992, no hay referencia directa al Estudio de Impacto Ambiental — supuestamente, base para realizar la Declaración-, salvo en una comparación de alternativas. Incluso en ese caso, no se explica la elección de la alternativa elegida como la menos mala, aun habiendo sido propuestas alternativas locales que se desestimaron.

- En muchos de los casos, las alternativas incluyen una duplicación de calzada en travesía que, en realidad, es una falsa alternativa, ya que el objetivo principal de la obra es precisamente evitar la travesía de población. Este constituye el único elemento, desde el punto de vista social, por el que se aceptan otras alternativas que pasan alejadas o por el exterior del casco urbano, sin valorar si con ello se han eliminado los niveles de ruidos admisibles u otros elementos socialmente relevantes.

- El resto de los impactos que se indican en las Declaraciones de Impacto Ambiental se reducen a: estimación de la ocupación de suelo de alta pro- 
ductividad agrícola, sin proponer medidas correctoras o compensatorias distintas de la expropiación o la recomendación vaga de evitar su ocupación, en la medida de lo posible; accesibilidad transversal mediante la reposición de pasos a distinto nivel sin valorar cuáles son los idóneos, ya que no todas las soluciones son correctas y, en todo caso, reducen la permeabilidad territorial; en algún caso se hace referencia a los planes territoriales y urbanísticos sin comprobar si el proyecto es compatible o no con ellos; con respecto a las vías pecuarias y yacimientos arqueológicos de interés, no siempre se consideran, y su sola mención no garantiza la reducción del impacto, posponiéndose para fases posteriores las soluciones de su corrección.

- En las Declaraciones de Impacto Ambiental de 1992 - 20 de las 33 emitidas - se observa un esfuerzo por explicitar el proceso de evaluación, incluyéndose un resumen de consultas y de participación pública, análisis del proyecto y análisis del contenido del EIA. Se hace una mayor referencia a la realidad concreta del ámbito del proyecto. Se continúa valorando escasamente la afección a suelos agrícolas y su impacto económico en la zona. Se insiste más en la accesibilidad del territorio, aunque no siempre se aportan soluciones concretas para que se reduzca el efecto barrera.

Se introduce, casi por primera vez, la referencia a la planificación urbana más allá de la reserva de suelo para la infraestructura proyectada realizada en tales planes. Sin embargo, no se analiza debidamente la interferencia de la nueva carretera con el planeamiento urbanístico y con los planes territoriales o sectoriales.

No se incorporan debidamente las consultas y alegaciones públicas, y no se da respuesta a las que suponen una alternativa a la solución adoptada.

Se siguen postergando medidas correctoras importantes en relación al ruido, ocupación de cultivos y accesibilidad territorial, incluso en el caso de proyectos de trazado para fases posteriores.

Se observa una tendencia a realizar el EIA y el período de información pública en las fases últimas del proyecto, cuando el diseño ya está desarrollado (7 de los 20 proyectos), impidiendo así la posibilidad de introducir soluciones alternativas.

Hasta ahí los datos relevantes, pero pongámoslo en un contexto más amplio.

Las carreteras se caracterizan por introducir variaciones significativas en la ordenación territorial y en la red viaria porque generan nuevas posibilidades de conexión, provocando la fluctuación en la demanda del suelo para usos comerciales, industriales, residenciales, siempre en detrimento del uso agrícola tradicional. Potencian, en general, una mayor movilidad, lo que genera a medio y largo plazo un desequilibrio entre la oferta y la demanda de las viviendas en las ciudades de origen y destino, al mismo tiempo que tienden éstas a absorber población desplazada de las poblaciones que han perdido accesibilidad relativa con la dinámica territorial generada. 
En general, pueden favorecer una mayor concentración de la población y la actividad económica en ciudades y en las inmediaciones de los nudos viarios importantes.

Conviene hacer previamente distinciones en cuanto al tipo de carretera e incluso en cuanto al tramo evaluado.

Las autopistas en España, en su gran mayoría, pretenden como objetivo principal un flujo alto de tráfico de largo recorrido y, por tanto, su trazado afecta de manera más evidente a la contaminación acústica y atmosférica de las poblaciones cercanas. Poseen un trazado más lineal que se integra peor en el entorno. Sus enlaces con la red viaria son más reducidos, por lo que afectan en menor medida a la red viaria comarcal en el sentido de mejorar su accesibilidad, aunque sí pueden verse afectadas en razón de su intercepción. A diferencia de otros lugares, en España las autopistas no se utilizan para desplazamientos cortos, porque en algunos casos es necesario pagar por su utilización, y tampoco benefician a la economía de la zona por la que pasan, ya que los viajeros no salen de ellas para demandar servicios (los servicios se resuelven en áreas de servicio en la misma autopista). En fase de construcción, estas obras pueden dar empleos locales y, durante su explotación, algunos puestos de trabajo en áreas de servicio.

Caben hacer para su análisis dos distinciones básicas de carreteras, además del trazado en autovía o carretera con un solo carril por sentido:

- Variante de población: desvía el tráfico de travesía, evitando riesgos de accidente, atascos, ruidos y contaminación atmosférica a la población, aunque supone una pérdida de negocio para los comercios del interior de la población. Está más ligada al planeamiento del suelo urbano y a la expansión del suelo urbanizable.

- Tramo de carretera entre ciudades o núcleos de población: atraviesa territorio no urbano, especialmente si es una carretera de nueva construcción, y afecta más comúnmente a espacios rurales o naturales. Está más ligado a la planificación de usos en suelo no urbanizable, con lo cual puede que aconseje la limitación de usos infraestructurales y sus edificaciones anejas.

Establecidas tales distinciones con respecto al proyecto y medio general afectado, se pasa a enumerar las consideraciones a tener en cuenta para el análisis del impacto social.

En primer lugar, convendría identificar los objetivos del proyecto y su inserción en la red viaria. Normalmente, las decisiones tomadas a este respecto pertenecen a un plan general o regional de carreteras, que justifica la necesidad del proyecto pero no su trazado, con lo que pueden hacerse, sin desviarse de tales objetivos, nuevas propuestas en caso de tratarse de estudio informativo.

Un segundo nivel sería valorar cómo va a verse modificada la red viaria comarcal y, con ello, el sistema de relaciones socioeconómicas entre núcleos. No hay que olvidar que las comarcas son unidades sociales y geográficas 
importantes en España. En el caso de una variante de carretera, es conveniente observar que normalmente se trata de un polo de servicios en el interior de la comarca y que a ella llegan otras carreteras importantes además de la que se proyecta, por lo que habría que analizar:

a) Las relaciones de dependencia económica y de servicios de su área de influencia, distinguiendo la dependencia directa de poblaciones o viviendas rurales en diseminado de las poblaciones o municipios más independientes.

b) El tráfico que se establece con motivo de relaciones comerciales que tiene su destino en esta ciudad y, por tanto, no va a dejar de entrar en la población, excepto si se le ponen dificultades de acceso. En este sentido, sería necesario estudiar la idoneidad de los enlaces proyectados.

En el caso de que no sea variante de población, se debería analizar el efecto que la carretera causará sobre las poblaciones que conecta y sobre el sistema de relaciones socioeconómicas entre poblaciones, para evaluar en qué medida se potenciarán unos y se relegarán otros. Un aspecto importante a cuidar son las relaciones entre poblaciones situadas a ambos lados del trazado de la carretera, por el efecto barrera que podría causarse entre ellas, con la definición de enlaces transversales o pasos a distinto nivel previstos.

En un tercer nivel deberían estudiarse los planes territoriales, sectoriales y urbanísticos, estos últimos referidos a los municipios en los que el proyecto ocupa parte del suelo. En este sentido, lo que interesa es valorar si el proyecto va a potenciar o perjudicar los objetivos de planificación y en qué medida pueden paliarse las contradicciones que se susciten. Si el proyecto introdujera inconvenientes insalvables, convendría estudiar soluciones alternativas al proyecto o, en su caso, a los planes vigentes.

Otro nivel importante de análisis son los usos del suelo ocupado por el proyecto. En el caso de los usos agrarios, habría que valorar su grado de explotación o abandono; calidad agrícola de los suelos; rendimiento; población que depende de esa actividad, constituyendo su único ingreso; tamaño de las parcelas y necesidad de reparcelación en aquellas parcelas que, por razón del proyecto, queden incomunicadas o expropiadas en parte; caminos de acceso a las parcelas y cortes producidos por la obra, etc. Se trata, en definitiva, de estimar si su impacto es significativo a nivel familiar y de la comunidad en su conjunto, y establecer medidas para evitar impactos negativos sobre los recursos y el modo de vida de la población, que van desde modificación del trazado hasta reparcelaciones o permutas de terreno, además de mantener el viario de acceso a las parcelas.

En el caso de los usos industriales, como se trata de suelo urbano, suele evitarse excepto que sea suelo urbanizable para uso industrial, en cuyo caso la corrección de impacto pasa por modificar ligeramente el trazado y propiciar un acceso a la carretera proyectada.

El suelo ocupado por servicios, en caso de duplicación de calzada, suele 
tener un impacto muy notable. Se debería evitar su ocupación, garantizando el acceso conveniente al trazado proyectado que permita la parada de viajeros. Pero debe evitarse la edificación futura e indiscriminada a ambos lados de la carretera para que no se convierta en una zona de expansión urbana, aumentando el riesgo de accidentes. En el caso de autovía, esto no suele ser posible, especialmente si se proyecta un vallado, pero se recomienda que el planeamiento municipal evite la expansión urbana paralela a la carretera.

Por último, en los usos del territorio para actividades culturales tradicionales, bien estén en la franja de ocupación de la carretera o se intercepte su campo visual o su acceso, se deberían valorar posibilidades alternativas de viario. Un ejemplo de tal impacto es, en la autovía de Andalucía, el corte del paseo de cipreses que conducía al cementerio de Santa Cruz de Mudela, que tradicionalmente ha sido frecuentado por los residentes en las tardes de verano.

No conviene olvidar, en el caso de las carreteras, la fragilidad de las zonas rurales, destacando cuando se trata de una nueva vía o cuando es variante de población en un entorno de población diseminado cuya actividad principal es agrícola. En ambos casos, la presión que los usos urbanos y terciarios ejercerán, una vez esté construida la carretera, puede provocar un abandono adicional de la actividad agrícola e incluso la emigración de los residentes que hayan trabajado en la construcción de la carretera, empleo por el cual pudieran haber dejado su actividad agrícola. No queremos descalificar con ello el nuevo desarrollo, sino llamar la atención sobre la importancia del conocimiento previo de su impacto en zonas frágiles.

En el caso de las variantes, su cerramiento ejerce un efecto barrera sobre los núcleos o viviendas rurales de su interior, desconectándolos del exterior y provocando, a largo plazo, la extensión del uso urbano hacia el borde de la variante. Este impacto es verdaderamente importante, ya que el descenso de la actividad agrícola en nuestro país se ha producido de forma acelerada, existiendo muy pocos incentivos al modo de vida rural, que se ve especialmente presionado en las inmediaciones de las ciudades. Además, los suelos de expansión urbana suelen ser los más fértiles.

La aceptación social del proyecto en este tipo de comunidades más vulnerables es imprescindible para detectar cambios que puede provocar el proyecto y para plantear correcciones adecuadas.

\section{CONTENIDO DE LOS ANALISIS DEL IMPACTO SOCIAL}

En general, los análisis del impacto social que se vienen haciendo en las EIA analizadas responden básicamente al modelo visto de las guías metodológicas. Cumplen, en sus mínimos, con los apartados que se supone tienen que cubrir, pero lo interesante es ver cómo lo cumplen.

Ya en sus formas se ha detectado que estos análisis, como ocurre igualmen- 
te con los del medio físico, incurren en situaciones como son falta de indicación de las fuentes de datos, utilización de datos atrasados aun habiéndolos más actuales, ámbitos de estudio no siempre significativos para el tema concreto que se está estudiando.

Son estudios básicamente descriptivos, con escaso análisis de diagnóstico y de previsión tentativa de evolución del medio —otros enfoques que significaran el planteamiento de objetivos sociales de futuro que pudieran ser referentes para el análisis de impacto ni se plantea su consideración-. Este hecho imposibilita tener una visión más global del medio, fundamental para la identificación y valoración de impactos.

Igualmente ocurre con los criterios, metodologías y técnicas para dicha identificación de impactos y para su valoración, que están ausentes para la mayor parte de ellos y, a veces, se hace de una forma muy general.

Lo más significativo, no obstante, es que, a pesar de que en mayor o menor medida se han cubierto los aspectos que típicamente se consideran para el medio social en la fase anterior de análisis del medio, cuando se trata de la identificación de impactos y de su valoración quedan extremadamente reducidos, y además a elementos que puedan ser fácilmente cuantificables. Algunos ejemplos típicos son el análisis del impacto del ruido y de la calidad del aire; la cuantificación de superficies, viviendas y otras pertenencias afectadas; el cálculo de ahorro de tiempo, en el caso de los proyectos de carreteras; o el empleo que se va a generar por razón del desarrollo del proyecto en cuestión.

Se detecta, por otra parte, una división absoluta entre la parte de análisis y valoración del medio físico y del medio social, lo cual revela una descoordinación y la ausencia de análisis interdisciplinar. Se concluye por lo general, en estos estudios, en la valoración de los efectos a través de una matriz de síntesis de impacto, que expresa en una columna los elementos del proyecto causantes del impacto y en la otra los elementos receptores del medio, y que caracteriza el impacto. Esta matriz expresa en bastante medida el carácter rígido y reducido que se le da a las valoraciones de impacto, utilizándose incluso, en bastantes casos, como instrumento de llegada en lugar de herramienta previa en la identificación de efectos.

Las medidas correctoras de los impactos son las únicas que finalmente quedan de los análisis del impacto ambiental. De esa manera, otro tipo de medidas complementarias, compensatorias y preventivas, que junto con las de tipo exclusivamente corrector conforman la filosofía de la evaluación ambiental, han quedado eliminadas. Pero incluso las medidas correctoras han tenido una consideración muy reducida. Así, por ejemplo, se han basado casi exclusivamente en aspectos del medio físico e incluso, dentro de éste, preferentemente en los aspectos de revegetación de tipo jardinería de las obras públicas, junto con algunas de compensación económica. De las medidas correctoras posibles, se consideran casi exclusivamente aquellas cuyos contenidos son fácilmente presupuestables y definibles en la memoria típica del proyecto. Esta situación implica, de hecho, una adaptación del resultado del Estudio de Impacto 
Ambiental al proyecto sometido a evaluación. Este hecho supone que se excluyen del control aspectos no directamente relacionados con la ejecución de obra, puesto que se trata de problemas no definidos previamente, o bien que por su naturaleza requieren una recogida de datos que permita evaluar impactos residuales y la aplicación de las medidas oportunas, pero tan importantes o más que éstos. Pero, es más, como no existe un control posterior de que se hayan llevado a cabo las medidas propuestas, es bastante probable que queden mermadas.

Esta falta de control es el resultado de las numerosas deficiencias encontradas en el procedimiento de EIA, que van desde el cumplimiento tan sólo relativo de dicho procedimiento por parte de la Administración; la aceptación de evaluar proyectos en cualquiera de sus fases de diseño, aunque sea ya la última, donde poco se puede modificar; la validación de proyectos cuyos Estudios de Impacto adolecen de informaciones o criterios decisivos y necesarios para la determinación de la significatividad de los impactos identificados; y, sobre todo, la emisión de Declaraciones de Impacto Ambiental basadas en un Condicionado ambiental que articula medidas correctoras que inciden tan sólo en los impactos de una forma reducida y, por ello, minimizando escasamente los efectos producidos por el proyecto en determinados elementos del medio. Están ausentes en ese Condicionado acciones, preventivas, compensatorias y correctoras de otros elementos del medio, fundamentalmente de tipo social, que no han sido evaluadas en su complejidad, estando muchas veces tales acciones fuera del alcance del contratista, sobre el que recae el grueso de las medidas medioambientales.

Finalmente, la EIA tiene que concluir en un programa de seguimiento y control de los impactos detectados y las medidas correctoras propuestas. Lo más significativo de estos programas es la falta de concreción en las acciones a llevar a cabo. En la mayoría de los casos, se limitan a plantear unos objetivos que inciden básicamente en el cumplimiento de las medidas correctoras, en especial aquellas ligadas a la integración paisajística de la misma (la revegetación principalmente, como se ha explicado anteriormente). Sin embargo, están prácticamente ausentes la verificación de la corrección de los impactos, la valoración de impactos residuales, la probabilidad de aparición de otros nuevos y, sobre todo, las posibilidades de poder articular nuevas medidas y quién es el responsable, en cada caso, del control y cumplimiento de los objetivos y planificación elaborados en el programa. En tales circunstancias, cabe señalar que no existe un Programa de Vigilancia y Control, ya que son tan poco efectivos como si no existieran.

El documento de síntesis del EIA es un requerimiento legal con la idea de que resuma, en un lenguaje comprensible para el público en general, el Estudio de Impacto Ambiental en todas sus fases. Sin embargo, en los estudios analizados nos encontramos con una falta de rigurosidad en este documento, produciéndose situaciones en que se incluyen solamente partes, no se indican los criterios de valoración de los impactos, o se hacen para el caso de los no signi- 
ficativos pero no para los perversos, ni se explican las matrices de síntesis de los impactos.

Lo anteriormente visto expresa la situación de los análisis del impacto social que se vienen haciendo en las EIA en la experiencia española. Pero veamos, aunque sea someramente, algunas de las características ${ }^{24}$ que, a nuestro entender, deberían tener estos estudios en cuanto a su contenido.

En general, hay bastante consenso en la comunidad de profesionales del campo del análisis del impacto social en las EIA en que éste tendría que abarcar los siguientes aspectos:

- la discusión de la acción o el proyecto propuesto, en cuanto a un análisis crítico de los objetivos que pretende y la posibilidad de alternativas, y también en los aspectos particulares del proyecto que pudieran ser la causa de impacto importante;

- el análisis de los componentes del medio ambiente social o humano susceptibles de verse afectados por el proyecto y por el impacto ecológico del mismo;

- los impactos probables de producirse (generalmente definidos como la diferencia entre la evolución futura de ese medio «con» la acción o el proyecto en cuestión y «sin» el proyecto), y

- las medidas que podrían tomarse para aprovechar las potencialidades positivas que se presentan y mitigar las negativas (evitándolas si es posible, en primer lugar, y minimizando su impacto, en su caso, y facilitando compensaciones por aquellos impactos negativos que no puedan ser evitados o corregidos).

El problema está en decidir qué impactos son los que pueden ocurrir con más probabilidad, qué factores hacen el impacto «significante», o qué se necesita considerar para decidir sobre los tipos de datos y análisis que son «viables» $\mathrm{y}$ «apropiados» para estos estudios.

Estas incertidumbres son lógicas, entre otras cosas, porque todavía queda mucho camino por recorrer en este campo de la evaluación ambiental, pero las cosas se complican cuando esas limitaciones en nuestros análisis —al igual que ocurre con los análisis biofísicos, por otra parte- se utilizan para recortar en la práctica el contenido de esos estudios. Gran parte de la problemática de este campo en España se deriva de la realización de estos estudios por profesionales sin formación en las ciencias sociales.

Siguiendo el proceso indicado, veamos en primer lugar algunos aspectos a destacar en relación con el análisis en detalle de la acción o proyecto a evaluar. En la medida en que el impacto va a ser el resultado de las características concretas del proyecto y de las características igualmente concretas del medio que

${ }^{24}$ Para más detalle, véase Mercedes PARDO, «La Evaluación de Impacto Ambiental: teorías, procesos, metodologías» (en prensa), 1994. 
va a afectar (un mismo proyecto puede producir impactos diferentes según el lugar donde se localice), es importante contar con información sobre el proyecto significativa y suficiente para el análisis de los cinco «sistemas» que normalmente se consideran ${ }^{25}$ : condiciones biofísicas y de salud, sistema territorial y usos del suelo, estructuras sociales, cultura y condiciones psicosociales, y tendencias económicas y demográficas; sin embargo, ése no es el caso la mayoría de las veces. Las memorias de los proyectos siguen siendo básicamente del mismo tipo que han sido tradicionalmente, y no tienen en cuenta las necesidades de información para la EIA. A veces ni siquiera se aporta un organigrama temporal y de la mano de obra que va a emplear.

Relacionado con lo anterior está el tema de la desagregación de fases del proyecto que sea significativa para el análisis del impacto social. Tradicionalmente se ha estudiado exclusivamente la fase del proyecto como tal, y la de construcción, basado en la asunción derivada de las ciencias físicas y biológicas de que ningún impacto tenía lugar antes del inicio de la construcción propiamente. Sin embargo, algunos de los impactos socioeconómicos más significativos no se producen solamente a través de los cambios físicos directos, sino por los significados socioeconómicos de dichos cambios — presente, pasado y futuro- y por la acumulación de experiencia a lo largo del tiempo. En general (Fraudenburg y Gramling, 1990), los seres humanos respondemos a lo que se ve como oportunidades y/o perjuicios, o más globalmente, al significado en nuestras vidas de la acción propuesta. Es por ello que interesaría ampliar las fases de análisis del proyecto al preproyecto, y también a la de final de vida para determinados tipos de proyectos (p. ej., una central térmica). De ello se deriva la necesidad de estudiar los efectos con perspectiva incluso generacional para determinados proyectos.

El segundo paso en las EIA sería la identificación del medio ambiente afectado. Ello debería hacerse sobre los «sistemas» arriba indicados, pero también sobre otros aspectos que precisamente tienen que ver con una concepción más integrada del medio ambiente como es especialmente la relación existente sociedad-medio biofísico local, en su evolución histórica. Por poner algunos ejemplos, sería importante que abarcara temas como los modelos de poblamiento en relación con las características físicas del territorio, el significado económico, recreativo, estético y otros del medio biofísico local. Habría que identificar áreas o recursos naturales importantes para la población afectada o subgrupos de ella, y en especial los usos - de consumo o no consumo- del medio biofísico que pueda ser afectado por la acción propuesta.

El tercer paso es la identificación y valoración de impactos. Temas como la magnitud de los impactos, su extensión y duración, su severidad, el carácter acumulativo, aun con las dificultades de la tarea y la falta de rigurosidad que se aplica a pesar del predominante enfoque tecnocrático, en cualquier caso se suelen tratar. Sin embargo, estas valoraciones deberían ampliarse a conocer el

${ }^{25}$ American Sociological Association, Comité Interprofesional, «SIA Standard», 1994. 
impacto diferencial en poblaciones que pudieran estar en mayor riesgo por razón de su mayor vulnerabilidad (niños, ancianos, sectores muy pobres, etc.), y también a los efectos distribucionales sociales de los impactos (es decir, cómo afectan de forma diferente a las distintas clases sociales).

Un aspecto general en el que habría que basar la valoración del impacto ambiental es en cómo puede afectar a la sostenibilidad ecológica y social del medio ambiente estudiado. Los criterios de un desarrollo que sea sostenible deberían ser un elemento importante de esa valoración ${ }^{26}$.

\section{CONCLUSIONES Y PROPUESTAS}

Este artículo pretende haber puesto de manifiesto la importancia de una conceptualización amplia e interrelacionada de medio ambiente en la definición del impacto social de proyectos en el marco de las EIA, desvelando los mecanismos de alteración que se producen en dos tipos de obras públicas en concreto - embalses y carreteras-, y mostrando que no se trata de procesos simples, sino que, por el contrario, exigen un desmenuzamiento de sus relaciones.

Del balance de los proyectos sujetos a Declaración de Impacto Ambiental a nivel nacional, hasta la fecha, pueden extraerse las siguientes conclusiones en relación con la mejora del procedimiento administrativo de la EIA:

- La predicción del impacto social es más efectiva si el Estudio de Impacto Ambiental se realiza en las fases preliminares de proyecto. Es más, incluso entonces, el nivel de eficiencia se ve muy reducido, ya que hay decisiones que se tienen que tomar en fases anteriores al proceso de planificación, por lo que debería realizarse Evaluación de Impacto Ambiental de políticas, planes y programas.

Un ejemplo claro referido a los casos estudiados son el Plan Nacional de Carreteras y el Plan Hidrológico Nacional, de los cuales hubiera sido positivo haber hecho la Evaluación de Impacto Ambiental correspondiente. Esa evaluación a ese nivel permite incorporar el concepto de medio ambiente y sus repercusiones sociales en la toma de decisiones desde sus inicios y, sobre todo, permite que se incorporen alternativas no previstas y que se evalúen los costes ambientales, en sentido amplio, desde el principio, rechazando soluciones que no son admisibles ecológica y socialmente.

La Evaluación de Impacto Ambiental aplicada a proyectos integrados en programas que han sido sometidos a EIA se situaría entonces en un nivel más

${ }^{26}$ Un planteamiento así está empezándose a plantear por organismos como el Banco Mundial para la evaluación medioambiental de sus proyectos de desarrollo, como ya se ha mencionado anteriormente. Para una crítica de lo que estas posiciones significan, véase Pérez Adán (1994). 
concreto, en el cual se habrían fijado ya los criterios generales de actuación. En la situación actual, sin embargo, cuando se comienza el proceso de evaluación medioambiental, el proyecto está demasiado definido, no permitiendo por ello plantear alternativas.

- Sería necesario retomar la consideración de que el Estudio de Impacto Ambiental evalúe la aceptación social del proyecto, tal y como reclama el RD 1131/88, y que se tenga el input de la participación pública desde las fases más incipientes del proyecto. Sería igualmente importante el cambio en el enfoque rígido de la participación pública, cuya base fundamental es la exposición pública del documento en los locales del Ayuntamiento y similares, a un enfoque mucho más abierto en donde, a través de formas participativas más dinámicas e interactivas, se incorpore la valoración social de proyectos y su negociación.

Un enfoque así complejiza el proceso, por supuesto, pero desde luego lo democratiza y, en última instancia, lo hace más eficaz en cuanto a la posibilidad real de detección de impactos, como ya se ha indicado con anterioridad, y en cuanto a la legitimación social de los proyectos de desarrollo. No es este último un enfoque ajeno, puesto que es moneda común en los países que más han desarrollado la Evaluación de Impacto Ambiental (Estados Unidos y Canadá), y sería necesario dar un cambio radical en la práctica que se lleva a cabo en nuestro país al respecto. No parece serio que se denomine participación pública a lo que se viene haciendo.

En concreto, se indican a continuación los objetivos principales que el procedimiento de la EIA debería contemplar en relación a la participación pública para su incorporación en las diferentes fases de la evaluación ambiental de proyectos.

Se plantean tres niveles de participación social:

\section{Nivel de viabilidad}

- Consultas previas: Selección de instituciones, colectivos y expertos que elaboren los criterios ambientales previos.

- Estudio de aceptación social: Sondeo de problemas y colectivos afectados y/o sujetos de opinión.

- Participación pública como tal: Diseño del procedimiento y consulta a los afectados y/o sujetos de opinión, y aportación de alternativas.

\section{Nivel de estudio de alternativas de localización o a la totalidad del proyecto}

- Consultas previas: Incorporación de otros a consultar con mayor experiencia/competencia en el territorio específico. Valoración ambiental con indicación de problemas específicos. 
- Estudio de aceptación social: Detección de los criterios para la negociación social en las distintas alternativas.

- Participación pública: Negociación de contrapartidas en las distintas alternativas.

\section{Nivel de desarrollo del proyecto}

- Consultas previas: Incorporación de otros sectores que hayan aparecido en el proceso. Valoración ambiental localizada.

- Estudio de aceptación social: Valoración de las pérdidas y daños por la implantación del proyecto.

- Proceso de participación pública: Negociación de cambios y compensaciones. Alegaciones.

- Se deberían realizar estudios de seguimiento para detectar impactos no identificados en el proceso de Evaluación del Impacto Ambiental y que revierten en una mejor previsión para otros proyectos y ámbitos similares.

Ello permitiría un avance teórico en el tema del impacto ambiental, su aplicación al campo concreto de la EIA, mayor conexión y beneficio mutuo entre la teoría y la práctica, y formación interdisciplinar, de manera que empecemos a crear las condiciones mínimas que nos capaciten algo más para abordar la complejidad de los problemas medioambientales a que nos estamos enfrentando.

Para finalizar, concluir que un proyecto de obra pública cualquiera proporciona beneficios a unos grupos sociales en detrimento de otros. La justificación social puede ser mayoritaria, pero la negociación refuerza los objetivos de beneficio social del proyecto, cuando disminuye los impactos. 


\section{REFERENCIAS BIBLIOGRAFICAS}

Allende LandA, José (1990): «La EIA marco de referencia y aspectos relevantes a debatir», Ciudad y Territorio, 83-1, pp. 45-66.

BENTON, Ted (1991): «Biology and Social Science: Why the return of the repressed should be given a (cautious) welcome», Sociology, vol. 25, núm. 1, 1: 29, febrero.

BISSET, R. (1987): «Post-Development Audits to Investigage the Accuracy of Environmental Impact Predictions", Zeitschrift für Umweltpolitik, 463-484.

Catton, William, y Dunlap, Riley E. (1978): «Paradigms, Theories and the Primacy of the HEP-NEP Distinction", The American Sociologist, 13: 256-259.

Clarke, Lee, y Short, James F. (1992): "Current Controversies in Social Studies of Risk", manuscrito para ser sometido a Annual Review of Sociology.

Culhane, P. J. (1987): "The Precision and Accuracy of U. S. Environmental Impact Statements", Environmental Monitoring and Assessment, 8: 217-238.

DALY, Herman E. (1991): Ecological economics and sustainable development: from concept to policy, World Bank, Washington, DC.

FreudenburG, William R., y Gramling, Robert (1990): «Community Impacts of Technological Change: Toward a Longitudinal Perspective», artículo presentado a la Rural Sociological Society.

LeLE, Sharachchandra (1988): «Sustainable Development: A Critical Review», World Development, vol. 19, núm. 6.

LÓPEZ, Vicente (1983): «La gestión medioambiental del ruido», INYPSA, no publicado.

Margalef, Ramón (1981): Perspectivas de la teoría ecológica, Barcelona, Blume (3.a ed.); edición previa en inglés en 1968.

Martínez Alier, Juan, y SChlupmann, Klaus (1987): Ecological economics: energy, environment and society, Oxford, Basil Blackwell.

Morin, Edgar (1983): El método. I. La naturaleza de la naturaleza. II. La vida de la vida. III. El conocimiento del conocimiento, Madrid, Cátedra.

Murdock, S. H.; Leistritiz, F. L.; Hamm, R. R., y Hwang, S. (1982): «An Assessment of Socioeconomic Assessments: Utility, Accuracy, and Policy Considerations», Environmental Impact Assessment Review.

NASH, Roderick (1989): The Rights of Nature, Madison, University of Wisconsin Press.

NorgaARD, Richard B. (1989): «Sustainability as Intergenerational Equity: The Challenge to Economic Thought and Practice», Environmental Impact Assessment Review, 44: 85-125.

PARDO, Mercedes (1987): "La situación de la Evaluación de Impacto Ambiental en España: problemas y oportunidades para la gestión medioambiental», Estudios Territoriales, núm. 25, septiembre-octubre.

Pardo, Mercedes; Fraguas, Alberto; Galindo, Pilar; González, Rafael; Ramos, Enrique, y Robledo, Cándido (1993): «Perspectivas de mejora de las EIA y el desarrollo sostenible», Fase I, Plan Regional de Investigación de la Comunidad Autónoma de Madrid. No publicado.

Pérez, José (1994): «En busca de un paradigma económico para la redefinición del concepto de desarrollo sostenible», trabajo presentado al XIII World Congress of Sociology.

Schnaiberg, Allan (1980): The Environment: From Surplus to Scarcity, New York, Oxford University Press.

TOBIAS, Michael (1984): Deep Ecology, San Marcos (California), Avant Books.

TOLBA, M. K. (1984): The premises for building a sustainable society —Address to the World Commission on Environment and Development, Nairobi, United Nations Environment Programme, octubre.

Toledo, Víctor M. (1991): «Modernidad y ecología: la nueva crisis planetaria», Ecología Política. 


\title{
RESUMEN
}

El análisis del impacto social de las obras públicas es parte integrante de las Evaluaciones de Impacto Ambiental (EIA) en España. En este artículo se analiza el impacto social a través de la investigación de las Declaraciones oficiales de Impacto Ambiental y situándolas en un marco más amplio relativo al tipo de conceptualización del impacto social que se hace en la legislación aplicable, en las guías metodológicas de análisis para este campo y en los estudios de impacto ambiental concretos. Se concluye con unas propuestas de integración de la participación pública en las diferentes fases de evaluación ambiental con objeto de su incorporación en el procedimiento administrativo legal de las EIA.

\begin{abstract}
In Spain, social impact assessment of projects in part of Environmental Impact Assessment (EIA). This paper analyzes the social impact of projects within the frame of EIA. The research is done on the official Environmental Impact Statements in a broader theoretical framework regarding the type of social impact conceptualization made by the legislation, the methodological guidebooks, and the specific environmental impact studies. It concludes with some proposals integrating public participation within the several environmental assessment phases to be included into the legal procedure of EIA.
\end{abstract}


TEXTO CLASICO 